\title{
Critical Limit Principle of Maximum Entropy and Reinterpretation of Scaling Laws
}

\author{
Yougang Feng \\ College of Physics, Guizhou University, Guiyang, China \\ Email: ygfeng45@aliyun.com
}

How to cite this paper: Feng, Y.G. (2018) Critical Limit Principle of Maximum Entropy and Reinterpretation of Scaling Laws. Journal of Modern Physics, 9, 2035-2044. https://doi.org/10.4236/jmp.2018.911128

Received: August 16, 2018

Accepted: September 11, 2018

Published: September 14, 2018

Copyright $\odot 2018$ by author and Scientific Research Publishing Inc. This work is licensed under the Creative Commons Attribution International License (CC BY 4.0).

http://creativecommons.org/licenses/by/4.0/

\section{c) (i) Open Access}

\begin{abstract}
The critical limit principle of maximum entropy is put forward, it's a sufficient condition to obtain accurate critical points, and ensure that the new phase system is still in the maximum entropy state. Two representations for the phase transition of Ising models are found; the universal formula of critical points is explained by thermodynamics. From the point of view of fractal geometry and the correspondence between symmetry and conservation, the scaling laws are reinterpreted. The self consistence equations for the universal class are set up, by which and the scaling laws higher accurate exponents to date are obtained. The temperature where the self similar transformation disappears is calculated.
\end{abstract}

\section{Keywords}

Entropy, Critical-Point, Scaling, Exponent, Self-Consistence

\section{Introduction}

As a fundamental issue, the critical phenomena theory has pervaded modern physics, the approach to the critical point, the critical fluctuation, and the critical exponents help us understand critical laws deeply. The precise calculation of critical points and critical exponents is the common goal of the researchers. The symmetry analysis that is usually applied in particle physics makes our research to a new state, and has got more quantitative results [1]. There are some important models, among which Ising model is particularly striking. However, the accurate evaluation of the critical points was always an unsolvable problem for $3 \mathrm{~d}$ models by conventional theory. The main difficulty is that one cannot get the exact value of a block spin because all conventional theories and methods are limited to Euclidean geometry. Euclidean geometry uses simple graphics as a tool 
to study certain objects with complexity. On the contrary, fractal geometry gets a simple result when using complicated self similar (SS) transformation. By means of fractal geometry, the accurate critical points for $2 \mathrm{~d}$ and $3 \mathrm{~d}$ models are obtained [2]. For an infinite lattice system, it seems that there are two ways to an ordered state: 1) In the fractal geometry sense, the local lattices are correlated to each other to form finite size block spins, and the system is ordered by infinite hierarchical SS transformation, i.e., the block side length can be considered as the lattice correlation length, and it is finite. 2) From the eyes of the Euclidean geometry, all lattices are correlated to one another to construct an ordered system, the correlation length is infinite. The system entropy in the first way is greater than that of the second way, since the ordering degree of the system in the first way is lower than that in the second way. In a word, relative to infinity, the finite correlation length makes the system more chaotic, thus the system entropy is greater.

The focus of both views is the lattice correlation length. Whether the correlation length is infinite or finite, we can respond in only one of the two ways. In fact, the concept of infinite correlation length is merely a theoretical assumption. We can't measure the infinite length; we can only measure the exponent $v$ that is associated with the length in term of the conventional theory. According to the assumption, the infinite correlation length occurs at the critical temperature $T_{c f}$, and disappears at the temperature $T$ that is in the vicinity of $T_{c f}$, and $T<T_{c f}$. The idea that the ordered state is derived from the lattice correlation is reasonable, but the argument that the correlation length disappears suddenly in the vicinity of $T_{c f}$ is hard to convince us. Let a system have $N_{m}$ lattices in total, a block contain $N$ lattices, the maximum hierarchy number of the SS transformation be $r_{m}$, we have

$$
r_{m}=\ln N_{m} / \ln N
$$

where the symbol "ln" represents natural logarithm. If the system is infinite, $N_{m} \rightarrow+\infty$, with the deceasing of temperature the lattice correlation length $n$ increases from $n^{*}$, which is the block side length at the critical pint, to infinity, so does $N$ at $T_{c s}$, where the correlation length becomes infinity and the whole system is a block [1]. Since $N$ is finite at the critical point, no matter what value $N$ takes, $r_{m}$ will tend to infinity due to the infinite $N_{m}$. However, this is an ideal situation that can't occur in nature. The exponent $v$ is a measurable parameter, and a specimen that can be measured only has limited size and lattices $N_{m}$, although it may be approximately considered as infinity. It is the finite $N_{m}$ that leads to that the maximum hierarchy number $r_{m}$ can't become infinity: When the temperature decreases after the transition, the correlation length (the block side length $n$ ) increases, and $r_{m}$ is changing smaller and smaller with $N$ is getting bigger and bigger. This proves that the exponent $v$ describes actually the behavior of the number $r_{m}$ rather than the correlation length's. For example, for the irreducible lattice system of Ising model [2], $r_{m}$ behaves as 


$$
r_{m} \propto\left|T-T_{c f}\right|^{-v}
$$

For the reducible lattice system [2], we have the similar conclusion.

This paper is formed by the above point of view. In Section 2, we put forward the critical limit principle of maximum entropy. We prove again the uniqueness of the critical point. From the dual relation of $J$ and $S^{2}$, we find two representations for the phase transition: spin representation and coupling constant representation. We clarify, in the thermodynamics sense, the meaning of the critical point. From the point of view of the SS transformation, we reinterpret the scaling laws that they are conserved laws corresponding to the symmetry transformation. We set up universal class self consistence constant and the relevant equations for the exponents, and point out that the exponents must satisfy simultaneously the scaling laws and the equations, and the scaling laws are available for the whole renormalization region $T_{c s}<T \leq T_{c f}$ rather than in the vicinity of $T_{c f}$. The structural meaning of the disorder-order phase transition is that the original lattice spin system has become the self-similar block spin system. In Section 3, we calculate $T_{c s}$ and exponents, get a group of exponents, which is the most accurate and self consistent datum to date. Section 4 is conclusion remark. For simplicity, both blocks and sub-blocks are uniformly called blocks in this paper, and the relation between the sub-block's dimension and the block's dimension for the reducible lattice system is given by the formula (6.2) of reference [2].

\section{Theory}

\subsection{Critical Limit Principle of Maximum Entropy}

The conventional theory thinks that since lattice spins are correlated over scales up to the correlation length $\zeta$, it may be plausible to regard the spins with regions up to $\zeta$ in size as behaving like a single block spin of side length $n$, and $n \leq \zeta$, i.e., the block formation originates from the infinite correlation length. It's said that one can get accurate critical point only if let $n$ take $\zeta$, if $\zeta \rightarrow+\infty$. In fact, this is not true [2].

We find that there exist simultaneously two sorts of blocks: $n_{+}$-blocks and $n_{-}$-blocks, only one of them can become ordered, another disordered [1]. So, there are two kinds of correlation lengths: the first refers to the ordered, the second to the disordered. They are different from each other, resulting in that both are finite. Consider a system consisting of infinite subsystems, the values of spins of the $\mathrm{i}$-th subsystem are identical and equal to $S_{i}$, the free energy is $F_{i}$; the system free energy $F$ is given by

$$
F=\sum_{i=1}^{\infty} P_{i} F_{i}
$$

where $P_{i}$ is the occurrence probability of the i-th subsystem. The value of $P_{i}$ can't change the influence of the singularity of $F_{i}$ on the singularity of $F$. If $P_{i}$ is determined by Gaussian distribution, $F$ is also the Gaussian distribution free energy, and represented in the form as the Equation (11) of reference [2]. The 
Gaussian distribution makes the system in the maximum entropy state [1]. We then get a principle:

Critical limit principle of maximum entropy: A thermodynamic equilibrium system keeps itself in the maximum entropy state before disorder-order phase transition, and tends to the critical point in this manner.

According to the fractal theory that a system containing a large plenty of lattice spins becomes ordered as the same as a point spin is a kind of spin contraction mapping, which ensures the uniqueness and existence of self similar transformation [3]. This principle pledges the uniqueness of the critical point. It is this principle that helps us get accurate critical points for $2 \mathrm{~d}$ and $3 \mathrm{~d}$ models [2]. It's should be emphasized that the Gaussian distribution differs from the Gaussian model, the former determine which subsystem is that we need; the latter allows the system change the value of the block spin at the same temperature, although all block spins have the identical value. The common feature of both systems is they have the same free energy singularity. This means that we can get the critical point by Gaussian model. Using the fixed-point Equation (5) of reference [1], we can prove that the block side length $n^{*}$ at the critical point is unique and finite. For the triangle lattice system, substituting $N=(n+1)(n+2) / 2$, see [2], into the equation:

$$
\left(2-D_{\min }\right) n^{2}+3\left(1-D_{\min }\right) n-2 D_{\min }=0
$$

Equation (4) has only a positive finite real solution $n^{*}$ due to $1<D_{\min }<2$. For the tetrahedron lattice system, $N=\left(n^{3}+6 n^{2}+11 n+6\right) / 6$, see [2], the equation becomes

$$
\left(3-D_{\min }\right) n^{3}+\left(12-6 D_{\min }\right) n^{2}+\left(11-11 D_{\min }\right) n-6 D_{\min }=0
$$

This equation has a unique finite real root $n^{*}$. As the same reason, there is a unique finite side length $n^{*}$ at the critical point for any one system, which accords with the result of numerical calculation [2].

\subsection{Dual Relation}

The energy conservation of transformation produces a dual relation of $J$ and $S^{2}$ [2]:

$$
Z J S^{2}=2 D j s^{2}
$$

where the lattice spin $s^{2}=1$, its coupling constant $j$, the block fractal dimension is $D$, its spin square is $S^{2}$, coupling constant $J$, and coordinate number $Z$. For a given system $Z$ is constant. Let $J=j$, we get spin representation:

$$
S^{2}=(2 D / Z) s^{2}
$$

If let $S^{2}=s^{2}$, we have coupling constant representation from Equation (6):

$$
J=(2 D / Z) j
$$

The two representations are equivalent. In the early renormalization group (RG) theory the coupling constant representation is adopted [4]. In the Gaussian 
distribution, we use the spin representation [2]. Both relate to the common transformation $(2 D / Z)$, and the fractal dimension $D$ takes the minimum $D_{\min }$ at the critical point. The theory that the critical point is a fixed point in the coupling constant representation should be equivalent to that the $D_{\min }$ at the critical point corresponds to the fixed point of the block side length in the spin representation as the two representations describe the same case.

\subsection{Universal Formula of Critical Points for Ising Models}

The singularity of the free energy gives rise to

$$
Z J S_{\min }^{2}=k_{B} T_{c f}
$$

This is a universal relation for the critical points of Ising models [2], where $T_{c f}$ is the disorder-order phase transition temperature, $k_{B}$ Boltzmann constant. At a temperature $T$, a system has a definite mean energy $\langle E\rangle$, its free energy $F$ and entropy $S_{e}$ obey thermodynamic condition:

$$
F=\langle E\rangle-T S_{e}
$$

Only if the entropy $S_{e}$ is maximum can the free energy $F$ be minimized when the $T$ is constant. That the block spin takes the minimum when the system reaches to $T_{c f}$ is the best selection to satisfy the condition. We may view $Z J S_{\min }^{2}$ as an ordering tendency, $k_{B} T_{c f}$ as a disordering tendency caused by thermal motion. The critical point not only is a balance point for the two tendencies, but also a starting point of the ordering of the system. The fact that the block spin formation is the self-organization behavior and the cooperation phenomena of the lattice spins. Discussing the atomic system, Dirac pointed out that the observed specific heats at ordinary temperatures are given fairly well by a theory that takes into account merely the motion of each atom as a whole and assigns no internal motion to it at all [5]. Likewise, we should only consider the interaction between the block spins, the interaction between the lattice spins exists only inside the block spins. Under the condition of the maximum system entropy, the increasing of the ordering degree is compensated by the decreasing of temperature, and the ordering tendency overwhelms the disordering tendency below $T_{c f}$ :

$$
Z J S^{2}>k_{B} T
$$

It's the lattice correlation length that determines the size of the block space, i.e., the block side length. The lower the temperature, the longer the correlation length, and the larger the block side length (the block spin) and the higher the ordering degree. By means of boundary condition, Onsager carried the plane square lattice system onto a torus, and obtained a lager critical point than ours [2] [6]: $0.4407>0.4387$, which means his model has lower critical temperature than the model on the plane. The lower critical temperature originates in the boundary condition, which requires an extra ordering degree, besides the plane systems. 


\subsection{Reinterpretation of Scaling Laws-Symmetry and Conservation}

The $T_{c f}$ is just the critical temperature $T_{c}$ defined by the conventional phase transition theory. We notice that there is no singularity behavior below $T_{c f}$, i.e., the block structure exists until $T_{c s}$ and the block spins replace the lattice spins. We call the region $T_{c s}<T \leq T_{c f}$ as renormalization region, rather than merely the criticality one.

The scaling laws are based on the scaling hypothesis, which is purely a conjecture. Taking Ising model as an example, we interpret it by Euclidean geometry and fractal geometry. From the point of view of Euclidean geometry, the reason why the GR theory works is that the lattice correlation length becomes infinity in the vicinity of $T_{c f}$, such that the influence of all finite microscopic characteristic lengths are wiped out, and the correlation length is a unique characteristic quantity. The singularities of thermodynamic parameters are attributed to the correlation length singularity, and any size transformation can't change the form of the free energy function, only changes the parameter scaling. On the other hand, the transformation hierarchy is emphasized in terms of fractal geometry: The formation of blocks with finite side length $n$ begins at $T_{c f}$, they can go through $r$-hierarchical SS transformations at the same temperature, and the original lattice spin system is replaced by the block spin system. For any finite value of $r$, one can find the same form of the free energy function represented by the block spins, no matter what value of $r$ will be. Clearly, both interpretations describe the same phenomena. It's impossible to explain in terms of Euclidean geometry the uniqueness of the block side length $n^{*}$ at the critical point and the coexistence of $n_{+}$-blocks and $n_{-}$-blocks. In fact, the renormalization transformation is just the self similar one. In a word, the geometrical structure and the physical parameters function forms on the $(r+1)$-th hierarchy maintain the original ones on the $r$-hierarchy. By the forms, we are unable to recognize which belong to the $r$-hierarchy and what is the actual value of $r$. The indistinguishability means symmetry [7]. The SS transformation comes essentially from the spacetime homogeneity and is a special type of symmetrical transformation, differing from the conventional operations such as rotation, translation, inversion, etc. The GR is a symmetry group, its corresponding conserved quantity is the scaling, and there exists scaling invariance. The scaling laws do be the conservation laws.

For a universal class of space dimension $d$ and order parameter dimension $m$, there are two independent conserved constants: $p$ and $q$, they can be considered as the magnitudes of two orthogonal constant vectors $\boldsymbol{p}$ and $\boldsymbol{q}$, respectively. According to Wilson theory [8], $p$ and $q$ are given by

$$
p \propto \ln (2 D / Z) / \ln N, q \propto \ln (\sqrt{2 D / Z}) / \ln N
$$

where the elementary excitation of block spins is badly neglected [9]. The actual relation between $p$ and $q$ should be as

$$
p=\sigma(d, m) q
$$

where $\sigma(d, m)$ is a universal class self consistence constant. Three parameters 
in this equation, only two of them are independent. $p$ and $q$ control six exponents:

$$
\begin{aligned}
& \alpha=2-1 / p, \quad \beta=(1-q) / p, \quad \gamma=(2 q-1) / p \\
& \delta=q /(1-q), \quad \eta=d(1-2 q)+2, \quad v=1 / p d
\end{aligned}
$$

They determine four scaling laws:

$$
\begin{gathered}
\alpha+2 \beta+\gamma=2, \quad \gamma=\beta(\delta-1) \\
\gamma=v(2-\eta), \quad v d=2-\alpha
\end{gathered}
$$

Using Equations (13)-(17), there should theoretically be 15 self consistence equations for $\sigma(d, m)$ by the combinations of any two different exponents. But, the combinations of $\alpha$ and $v, \eta$ and $\delta$ have no consequence, 13 equations are actually established:

$$
\begin{gathered}
\sigma_{1}=1 /(2-\alpha-\beta), \quad \sigma_{2}=1 /(\beta+\gamma), \quad \sigma_{3}=2 d /[(2-\alpha)(2+d-\eta)] \\
\sigma_{4}=(\delta+1) / \delta(2-\alpha), \quad \sigma_{5}=2 /(\gamma+2-\alpha), \\
\sigma_{6}=1 /(\beta \delta), \quad \sigma_{7}=1 /(d v-\beta), \quad \sigma_{8}=(\delta-1) /(\delta \gamma), \quad \sigma_{9}=2 /(\gamma+d v) \\
\sigma_{10}=(4-2 \eta) / \gamma(d+2-\eta), \quad \sigma_{11}=2 d /[v d(d+2-\eta)] \\
\sigma_{12}=(1+\delta) /(v d \delta), \quad \sigma_{13}=(d-2+\eta) / \beta(d+2-\eta)
\end{gathered}
$$

The conservation requires that all exponents should not only obey the scaling laws, but also satisfy the self consistence equations, and $\sigma_{i}=\sigma(d, m)$ for all $i$, $i=1,2, \cdots, 13$. Our experience indicates that the scaling laws are not sensitive to the errors of the exponents: if the deviation between the exponents values and a law is 0.0001 , the difference between the high accurate value of the exponent and its crude value is usually greater than 0.0001 . As this reason, the exponents must strictly obey the laws. In most cases, the self consistence equations have higher sensitivity to the exponents values: the exponents that can comply with the laws are not necessarily accord with them. Only those exponents that satisfy both the laws and the self consistence equations have higher accuracy.

The experimental datum illustrate that all order parameters curves are smoothly continuous when the temperature is below $T_{c f}$ [8]. We think that the scaling laws and the self consistence equations are applicable to the renormalization region $T_{c s}<T \leq T_{c f}$, and the exponents values are determined by $T$. The relevant power law can be written as

$$
\left(T_{y}-T\right)^{ \pm x}
$$

where $T_{y}$ is in the normalization region, $T_{y}>T$, and $T$ in the vicinity of $T_{y}$, $x=\alpha, \beta, \gamma, \delta, \eta, v$, their positive and negative signs are the same as the ones' at $T_{c f-0}$, which is in the vicinity of $T_{c f}$, and $T_{c f-0}<T_{c f}$. For example, by Equations $((1),(2)$, and (23)), we get

$$
\ln N \propto\left(\ln N_{m}\right)\left(T_{y}-T\right)^{v}
$$


$N$ increases with the decreasing of $T$. Equation (24) verifies that the power law is a characteristic of fractals [10].

\section{Discussion}

\subsection{Calculation of $T_{c s}$}

Whether it is irreducible system or reducible system [2], the blocks disappear at $T_{c s}$, where the mean energy $\langle E\rangle$ of the elementary excitation of the block spins vanishes. We then get the following consequence [9]

$$
\langle E\rangle=\int_{0}^{x_{D}} \frac{x^{2}}{\mathrm{e}^{x}-1} \mathrm{~d} x=0
$$

where $x=\hbar \omega / k_{B} T, x_{D}=\hbar \omega_{D} / k_{B} T_{c s}, \hbar$ is Plank constant, $\omega$ the magnetic phonon frequency, $\omega_{D}$ the Debye frequency. Generally, we may get $T_{c s}$ from the integral equation. If $\mathrm{e}^{x} \gg 1, \mathrm{e}^{x}-1 \approx \mathrm{e}^{x}$. Equation (25) simplifies as

$$
\int_{0}^{x_{D}} x^{2} \mathrm{e}^{-x} \mathrm{~d} x=0
$$

This leads to an algebraic equation

$$
x_{D}^{2} \mathrm{e}^{-x_{D}}+2 x_{D} \mathrm{e}^{-x_{D}}+2 \mathrm{e}^{-x_{D}}-2=0
$$

We'll get $T_{c s}$ from the solution. If $\mathrm{e}^{x} \approx 1$, Equation (25) becomes

$$
\int_{0}^{x_{D}} x \mathrm{~d} x=0
$$

$x_{D}=0$, the solution is unreasonable.

\subsection{Numerical Estimation of Critical Exponents}

The elementary excitation of block spins also contributes to the free energy nonsingularity [9]. In addition, there is disorder-order state transformation between the $n_{+}$-blocks and the $n_{-}$-blocks [2], all these add the complexity of calculating the exponents. Usually, they are obtained by numerical approximation, in which process it's common practice to introduce appropriate adjustable parameters. The usual methods involve $\varepsilon$-expansion, field theory, Monte Carlo, high-temperature expansion, etc. [11]. A number of expansions are divergent, for example, the $\mathcal{E}$-expansion. An experienced author can get more exact results from the series by using Borel summation and educated guess. In this regard, Zinn-Justin achieved success [12]. Unfortunately, we examine that his exponents can't satisfy simultaneously the scaling laws and the self consistence equations. The self consistence equations provide us a new key for the numerical simulation. Based on the datum given by him and others [8] [11] [12] [13] [14], we get higher accurate solutions by golden section method and numerical approximation in the scaling laws and the self consistence equations. They are listed in the Table 1 below, including the exponents of $2 \mathrm{~d}$ Ising model. Because all exponents are dependent on the constant $\sigma(d, m)$, the repetitive operation may produce an extra additive effect of errors. In order to reduce the superposition, we make the $p, q$, and the constant $\sigma(d, m)$ accurate to the sixth decimal place, the exponents and 
Table 1. Universal class self consistence constant and critical exponents.

\begin{tabular}{cccccccccc}
\hline$d, m$ & $\sigma(d, m)$ & $q$ & $p$ & $\alpha$ & $\beta$ & $\gamma$ & $\delta$ & $\eta$ & $v$ \\
\hline 2.1 & $8 / 15$ & $15 / 16$ & $1 / 2$ & 0 & $1 / 8$ & $7 / 4$ & 15 & $1 / 4$ & 1 \\
3.1 & 0.638613 & 0.827204 & 0.528263 & 0.1070 & 0.3271 & 1.2388 & 4.7872 & 0.0368 & 0.6310 \\
3.2 & 0.610231 & 0.817227 & 0.498697 & -0.0052 & 0.3665 & 1.2722 & 4.4713 & 0.0966 & 0.6684 \\
3.3 & 0.583511 & 0.826243 & 0.482122 & -0.0742 & 0.3604 & 1.3534 & 4.7552 & 0.0425 & 0.6914 \\
\hline
\end{tabular}

constant $\sigma_{i}$ accurate to the fourth decimal place, so as to let the extra errors lie as behind as possibly the fourth decimal point. In principle, if we want the exponents and the constant $\sigma_{i}$ to be accurate to the x-th decimal place, the constant $\sigma(d, m), p$, and $q$ should be to the $(\mathrm{x}+2)$-th decimal place. For example, the following exponents and $\sigma_{i}$ for the universal class of $d=3$ and $m=1$ are accurate to the sixth decimal point, $x=6: \alpha=0.107063, \beta=0.327093$, $\gamma=1.238571, \delta=4.787153, \eta=0.036779, v=0.630979$, $\sigma_{i}=0.638633(i=1,2, \cdots, 13)$; and the others to the eighth decimal place: $\sigma(3,1)=0.63863311, p=0.52827948, q=0.82720347$. This group of exponents is the most accurate and self consistent datum to date. More accurate exponents will be obtained by the same method.

\section{Conclusion Remark}

The critical limit principle of maximum entropy introduces a correct way to obtain accurate critical points. The reinterpretation of the scaling laws from the correspondence between symmetry and conservation gives us a new understanding of scaling invariance. Based on the experimental and theoretical datum given by other authors, and applying golden section method and numerical approximation in the scaling laws and the self consistence equations, we can get higher accurate exponents.

\section{Conflicts of Interest}

The author declares no conflicts of interest regarding the publication of this paper.

\section{References}

[1] Feng, Y.G. (2018) Journal of Modern Physics, 9, 241-249. https://doi.org/10.4236/jmp.2018.92016

[2] Feng, Y.G. (2014) American Journal of Modern Physics, 3, 184-194.

[3] Kigami, J. (2001) Analysis on Fractals. Cambridge University Press, Cambridge. https://doi.org/10.1017/CBO9780511470943

[4] Domb, C. and Green, M.S. (1976) Phase Transitions and Critical Phenomena, Vol. 6. Academic Press, London.

[5] Dirac, P.A.M. (1958) The Principles of Quantum Mechanics. 4th Edition, Oxford University Press, Oxford.

[6] Onsager, L. (1944) Physical Review, 65, 117. https://doi.org/10.1103/PhysRev.65.117 
[7] Weyl, H. (1952), Symmetry. Princeton University Press, New Jersey. https://doi.org/10.1515/9781400874347

[8] Ma, S.-K. (1976) Modern Theory of Critical Phenomena. W. A. Benjamin, Inc., Massachusetts.

[9] Feng, Y.G. (2014) American Journal of Physics, 3, 211-217.

[10] Bak, P., Tang, C. and Wiesenfeld, K. (1987) Physical Review Letters, 59, 381-383. https://doi.org/10.1103/PhysRevLett.59.381

[11] Binney, J.J., Dowrick, N.J., Fisher, A.J. and Newman, M.J. (1992) The Theory of Critical Phenomena: An Introduction to the Renormalization Group. Oxford University Press, Oxford.

[12] Zinn-Justin, J. (2007) Phase Transitions and Renormalization Group. Oxford University Press, Oxford. https://doi.org/10.1093/acprof:oso/9780199227198.001.0001

[13] Moshe, M. and Zinn-Justin, J. (2003) Physics Reports, 385, 69-228. https://doi.org/10.1016/S0370-1573(03)00263-1

[14] Pelissetto, A. and Vicari, E. (2002) Physics Reports, 368, 549-727. https://doi.org/10.1016/S0370-1573(02)00219-3 